\title{
A Unique Spectrum of Tuberculosis Flare Up Related to Cardiothoracic Surgery
}

\author{
Khaled E. Al-Ebrahim, FRCSC \\ Department of Cardiothoracic Surgery, King Abdulaziz University, Jeddah, Saudi Arabia
}

\section{ABSTRACT}

Three cases of tuberculosis (TB) related to or complicating cardiothoracic surgery are presented in this paper. The aim of this article is to alert cardiothoracic surgeons about the presence or rebound of TB, which can complicate cardiothoracic surgeries even in the immediate postoperative course.

\section{INTRODUCTION}

Tuberculosis $(\mathrm{TB})$ is one of the most destructive chronic inflammatory diseases. Despite efforts of world health organizations, TB still is present and even endemic in some parts of the world. Factors leading to aggressive rebound of TB include lack of vaccinations and proper screening, incomplete treatment, and noncompliance of the patients.

\section{PATIENTS AND METHODS}

Three cases of destructive tuberculosis related to or complicating cardiothoracic surgery in different presentations are shown in this report.

Case No. 1 is a 45-year-old male, who presented with recurrent hemoptysis 2 years post-resection of a huge thymolipoma, weighing more than 5 kilograms (Figure 1). All of his old chest $\mathrm{x}$-rays were normal. Repeat chest $\mathrm{x}$-ray in the emergency room and computerized tomography showed bilateral lung destruction, more on the left lung (Figure 2). Acid-fast bacilli were isolated, and the patient was started on anti-tuberculous medications.

Case No. 2 is a fulminant flare up of tuberculosis on the second day post-transatrial repair tetralogy of Fallot on a previously healthy 10-year-old child. The ventricular septal defect was patched; the right ventricular outflow tract obstruction was relieved by muscle resection. There was acceptable transpulmonary gradient. Immediate postoperative course was smooth with good oxygenation and minimal inotropes. On the second postoperative day, the right lower lobe was opacified with deterioration in ventilation and oxygenation. The consolidation dramatically disseminated and

Received December 23, 2019; accepted fanuary 30, 2020.

Correspondence: Khaled E. Al-Ebrahim, Department of Surgery, King Abdulaziz University Hospital, King Abdulaziz University, P.O. Box 80215, feddah 21589, Saudi Arabia; fax: 966126401238 ; 966126401000 (e-mail: Dr.k.ebrahim@gmail.com). spread to both lung fields with pneumomediastinum and subcutaneous emphysema (Figure 3). This required prolonged ventilation. Molecular mycobacterium tuberculosis complex was detected, and the child was started on an anti-tuberculous regimen with good response and improvement of the pulmonary infection. Following 4 weeks of ventilation, he was extubated and discharged well.

Case No. 3 is a 15 -year-old cachectic female, who presented with multiple sternal discharging sinuses and back pain. Pus discharge culture grew mycobacterium tuberculosis. Computerized chest scan showed complete sternal destruction and paraspinal abscess (Figure 4). Open drainage of the sternal abscess and percutaneous drainage of the paraspinal abscess was done. The patient was started on anti-TB medications. The sternum healed by fibrosis following 6 months of treatment.

\section{DISCUSSION}

We report 2 cases of fatal tuberculosis (TB) post-cardiothoracic surgery. Both patients had no history of active TB. This usually is due to reactivation of dormant $\mathrm{TB}$, during depressed cellular immunity. The interval between surgery and appearance of TB varied in both cases. The first was 2 years post-thymectomy, which may have decreased the cellular immunity and lead to a TB infection. The second case was fulminant flare up of TB immediately post surgery and thought to be a reactivation of previous infection. The generalized inflammatory response associated with cardiopulmonary bypass $(\mathrm{CPB})$ affects patient immunity, predisposing the patients to infection. Transient immunodeficiency is known to occur after $\mathrm{CPB}$, as it affects the immune systems leading to qualitative and quantitative changes. There is a decrease in $\mathrm{CD} 3+, \mathrm{CD} 4+$ and $\mathrm{CD} 8+\mathrm{T}$ cells with a reversal of the normal CD4+/CD8+ ratio. Such changes are maximal on postoperative Day 1 and remain for approximately 1 week. TH lymphocytes is severely suppressed beginning on postoperative Day 1 [Lante 2005].

Reviewing the literature, this is the most acute postoperative TB infection in the intensive care unit. Both cases responded well to anti-tuberculous treatment. Rupali and colleagues [Rupali 2008] reported 5 cases of granulomatous hepatitis, following cardiopulmonary bypass and found that TB was reactivated from a dormant focus due to a period of transient immunodeficiency caused by extracorporeal circulation. Constrictive pericarditis and sternal tuberculosis post-cardiac surgery also were reported [Khalil 2006; Gopal 2007]. 

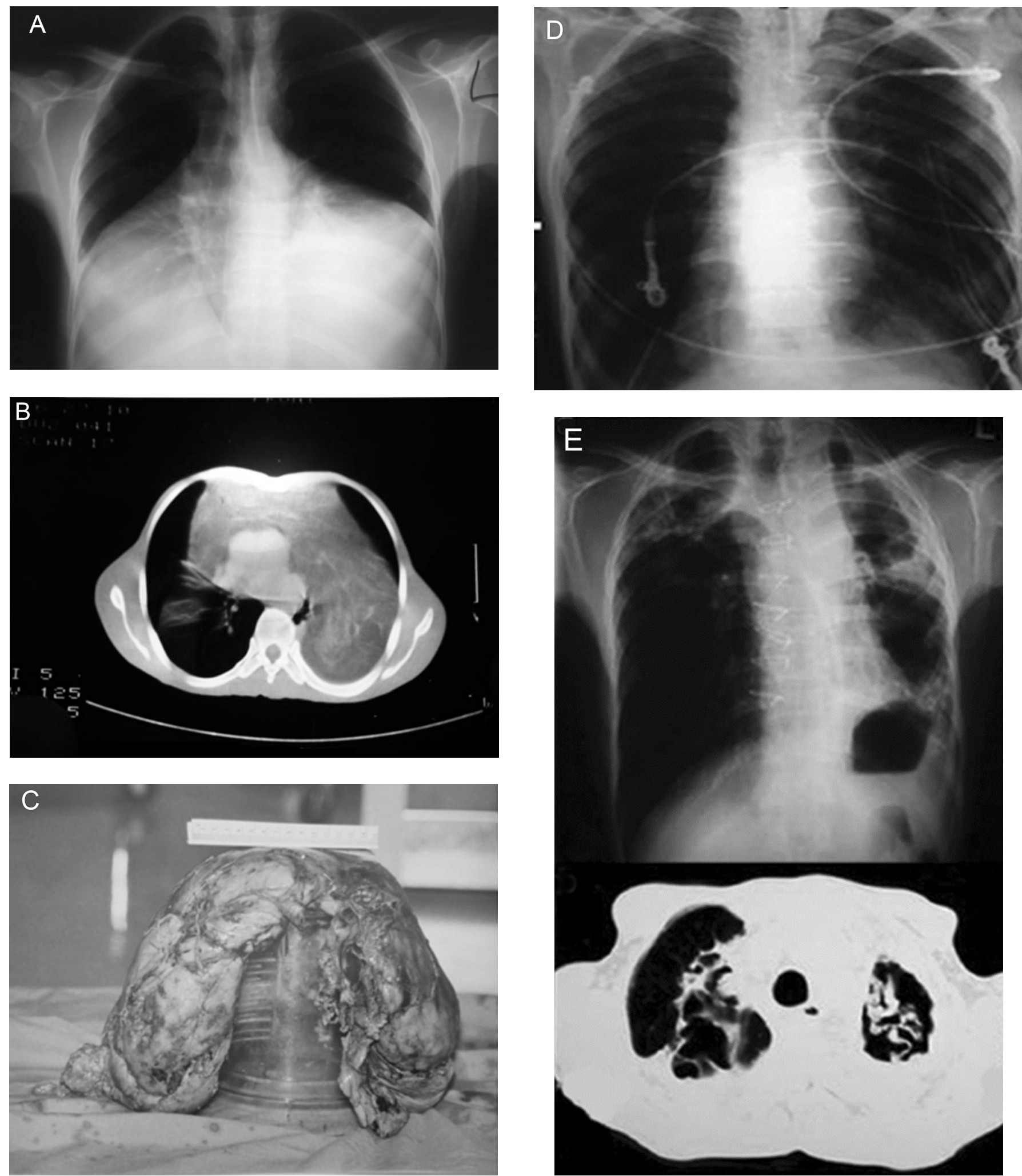

Figure 1. A, preoperative chest $x$-ray showing huge thymolipoma simulating cardiomegaly. B, Chest CT scan showing giant thymolipoma encasing normal-sized heart. C, Thymolipma weighing more than 5 kilograms. D. Postoperative chest x-ray showing normal lungs. E. One-year post-resection of thymolipoma chest $\mathrm{x}$-ray and $\mathrm{CT}$ scan showing left lung destruction and emphysematous right lung. 
TB of the sternum is rare. Up to one-third of such patients had a detectable tuberculosis lesion in other parts of the skeleton or in the lungs. Surgical treatment for tuberculous osteomyelitis of the sternum involves extensive debridement and curettage of all involved tissue. The defect can be closed primarily if possible or by mobilization of the overlying pectoralis major as a flap. Other methods described in the literature include vacuum-assisted closure with a drain or use of a pedicled omental flap to help close the defect [Tuli 2000]. The possibility of sternal TB should be kept in mind in any patient with a nonhealing ulcer or abscess in the sternal region. Early diagnosis and detection of mycobacterium tuberculosis by polymerase chain reaction provides a faster and reliable method for diagnosis to start anti-TB treatment as soon as possible in those critical conditions. Diagnosis usually is made by culture of the sputum, tracheal aspirate, discharge or the
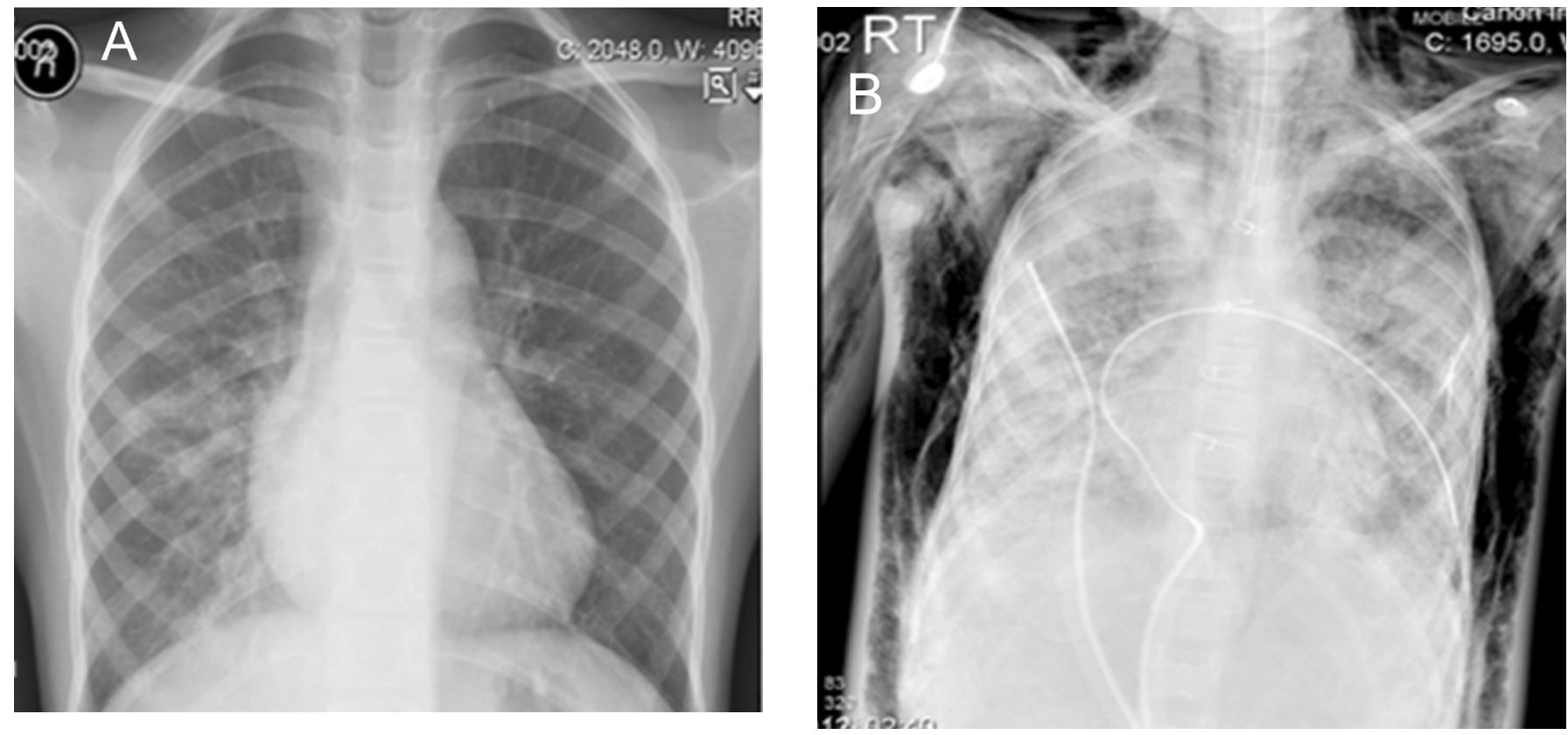

Figure 2. A, Preoperative chest $x$-ray, which appears to be normal. B, Postoperative chest $x$-ray, showing bilateral pulmonary congestion which progressed over days later to subcutaneous emphysema.
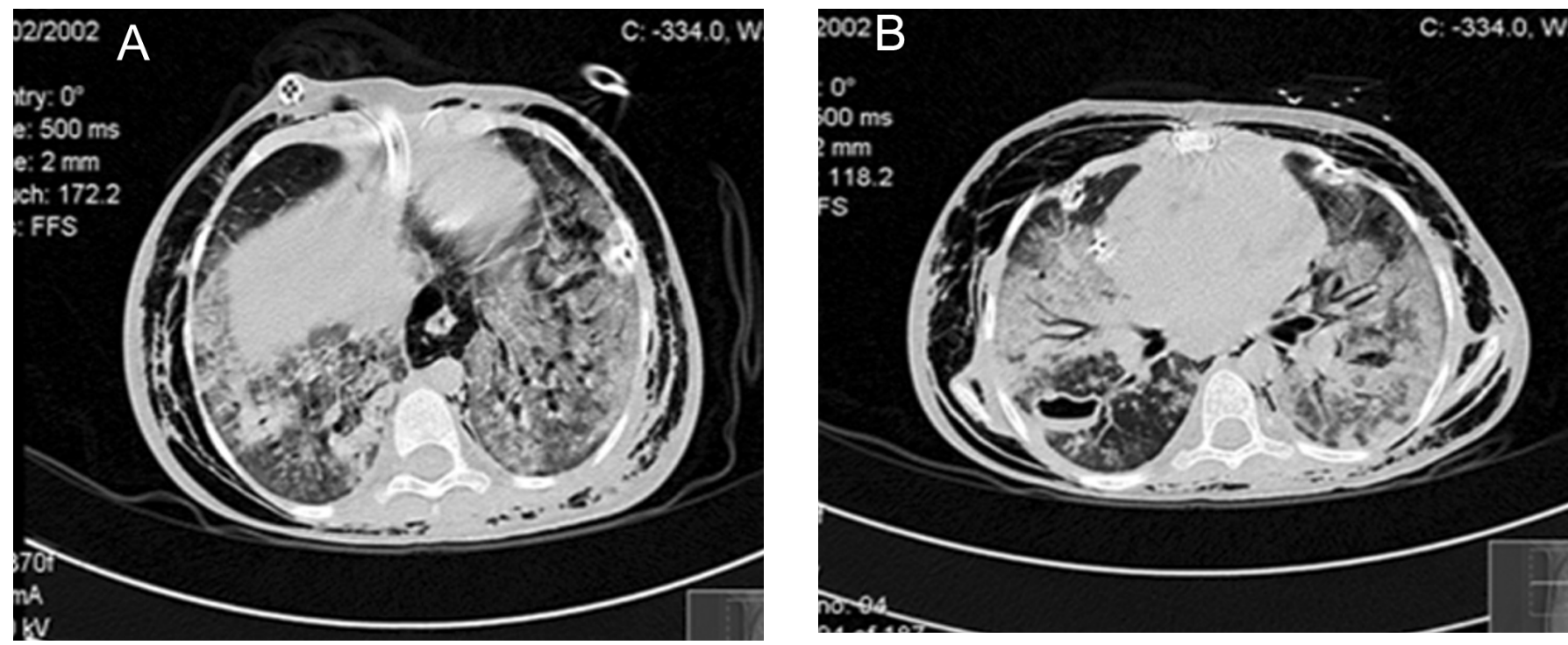

Figure 3. A, Postoperative CT scan, showing lung destruction mainly the left lung. B. Postoperative CT scan, showing multiple cavitating lesions more on the left lung. 


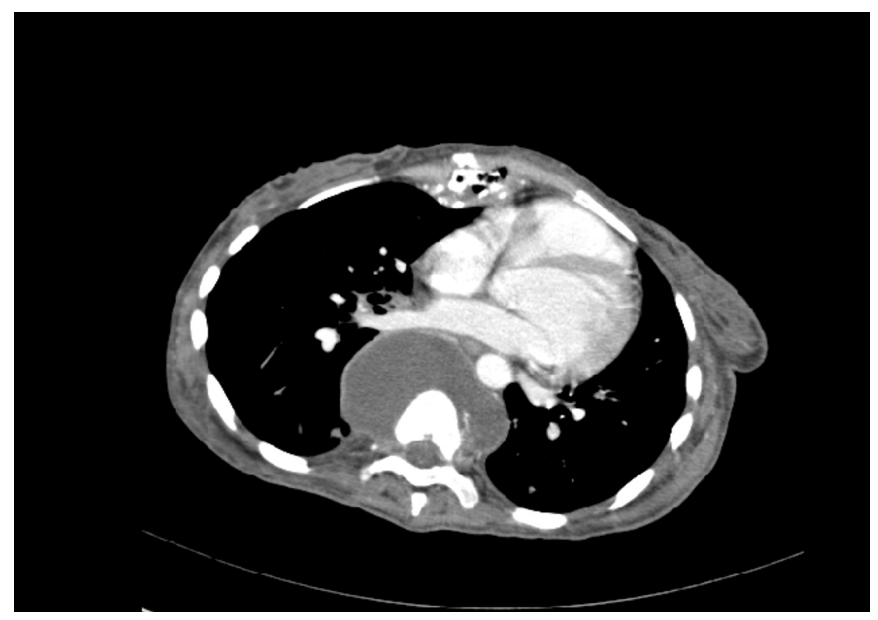

Figure 4. Computerized chest scan showing complete sternal destruction and huge paraspinal abscess.

resected tissue or by histopathologic examination. Initial culture reports may be negative, and in cases with recurrent non-healing wounds, repeat cultures for TB should be advised. In patients who come from areas where TB is endemic, a full assessment, including a Heaf test reaction should be carried out during preoperative assessment.

\section{ACKNOWLEDGEMENT}

This project was funded by the Deanship of Scientific Research (DSR), King Abdulaziz University, Jeddah, Saudi Arabia, under grant No. (1441-76-140). The authors therefore, gratefully acknowledge the DSR technical and financial support.

\section{REFERENCES}

Gopal K, Raj A, Ragesh M, Prabhu S, Geothe J. 2007. Sternal tuberculosis after sternotomy for coronary artery bypass surgery: A case report and review of the literature. J Thorac Cardiovasc Surg $133: 1365-6$

Khalil MW, Grech ED, Al-Mohammed A, Cooper GJ, Sarkar PK. 2006. Rapid development of fatal TB constrictive pericarditis after cardiac surgery. J Card Surg 21(4):417-8.

Lante W, Franke A, Weinhold C, Markewitz A. 2005. Immunoglobulin levels and lymphocyte subsets following cardiac operations: Further evidence for a T-helper cell shifting. Thorac Cardiovasc Surg 53:16-22.

Rupali R, Evangelynn S, Abraham O, Korola R, Shukla V, Thankachen R, Ponniah M, Subramanian S, Jeyaseelan V, Mathal D. 2008. Granulomatous hepatitis following open heart surgery with cardiopulmonary bypass. National Medical Journal of India 21(5):222-4.

Tuli SM. 2000. Tuberculosis of rare sites, girdle and flat bones. In: Tuli SM, editor. Tuberculosis of the Skeletal System (Bones, Joints, Spine and Bursal Sheaths). 2nd ed. Delhi: Jaypee Brothers Medical Publishers (P) Ltd pp. 159-160. 